\title{
ON THE FORMULATION OF BASAL DEBRIS DRAG FOR THE CASE OF SPARSE DEBRIS
}

\author{
By E.M. SHoemaker
}

(Faculty of Science, Simon Fraser University, Burnaby, British Columbia V5A 1S6, Canada)

\begin{abstract}
Two models are presented for the formulation of abrasion and basal drag due to rock-rock friction (debris drag) for the case of sparse debris entrained in the basal layers of a temperate glacier resting on a bedrock bed. The first model is formulated in terms of average basal melting rate, $v_{\mathrm{a}}$, and the concentration, $C$, of basal debris fragments which make intermittent bed contact. The second model is formulated in terms of $v_{\mathrm{n}}$, the component of ice velocity normal to the bed flowing around rock fragments contacting the bed, and $C_{\mathrm{c}}$, the concentration of debris actually in contact with the bed The relationship between the two models is given for the case of a sinusoidal bed. Generalizations are discussed as well as potentially important physical processes which remain to be investigated.
\end{abstract}

\section{LIST OF SYMBOLS}

This list does not include symbols which are only used where they are first defined.

\begin{tabular}{|c|c|}
\hline$a$ & Amplitude of sinusoidal bed \\
\hline$\dot{a}$ & Local abrasion rate \\
\hline$\dot{A}$ & $\begin{array}{l}\text { Abrasion rate averaged over a bed } \\
\text { wavelength }\end{array}$ \\
\hline C & $\begin{array}{l}\text { Concentration (projected area/unit area) } \\
\text { of local debris which makes intermittent } \\
\text { bedrock contact }\end{array}$ \\
\hline$C_{\mathrm{c}}$ & $\begin{array}{l}\text { Debris concentration which is actually } \\
\text { in bedrock contact averaged over time } \\
\Delta t=\ell / u_{\mathrm{b}} \text {. See Equation (17) }\end{array}$ \\
\hline$d$ & $\begin{array}{l}\text { Spacing of spherical debris fragments in } \\
\text { square array }\end{array}$ \\
\hline$F$ & Rock-rock contact force normal to bed \\
\hline$k$ & $k=2 \pi / l$ \\
\hline$\ell$ & Wavelength of sinusoidal bed \\
\hline$R$ & Radius of spherical debris fragments \\
\hline$R *$ & $\begin{array}{l}\text { A transition radius } \approx 0.1 \mathrm{~m} \text { if } n= \\
3 \times 10^{12} \mathrm{~Pa} \mathrm{~s}\end{array}$ \\
\hline$u_{\mathrm{b}}$ & Basal sliding velocity \\
\hline$v_{\mathrm{a}}$ & $\begin{array}{l}\text { Average basal melting rate at a point } \\
\text { on the bed as the point travels } \\
\text { distance } \ell \text {. }\end{array}$ \\
\hline$v_{\mathrm{g}}$ & Basal melting rate due to geothermal heat \\
\hline$v_{\mathrm{n}}^{5}$ & $\begin{array}{l}\text { Component of ice velocity normal to } \\
\text { the bed }\end{array}$ \\
\hline$v_{\mathrm{m}}(x)$ & $\begin{array}{l}\text { Basal melting rate with regelation } \\
\text { neglected }\end{array}$ \\
\hline $\bar{v}_{\mathrm{nc}}$ & $\begin{array}{l}\text { Average of } v_{\mathrm{n}} \text { over interval } x_{1} x_{2} \text { of } \\
\text { rock-rock contact }\end{array}$ \\
\hline $\bar{v}_{\mathrm{ns}}$ & Average of $v_{n}$ over stoss surface \\
\hline$x, x_{1}, x_{2}, y$ & Defined in Figure 1 \\
\hline $\begin{array}{l}\hat{n}_{\mathrm{R}} \\
\tau_{\mathrm{b}}\end{array}$ & $\begin{array}{l}\text { Defined by Equation (3) } \\
\text { Basal shear stress averaged } \\
\text { wavelength } \ell\end{array}$ \\
\hline$\tau_{\mathrm{d}}$ & $\begin{array}{l}\text { Debris-drag stress averaged } \\
\text { wavelength } \ell, \quad \text { computed from models } \\
\text { developed in paper }\end{array}$ \\
\hline$\tau_{d \mathrm{H}}$ & $\begin{array}{l}\text { Debris-drag stress computed from Hallet's } \\
\text { model }\end{array}$ \\
\hline$n$ & Viscosity of basal ice \\
\hline (n) & Coefficient of rock-rock friction \\
\hline
\end{tabular}

\section{INTRODUCTION}

An analysis of the basal shear stress (debris drag) and abrasion produced by occasional rock fragments (sparse debris) entrained in the basal layer of temperate glacier ice was given by Hallet $(1979,1981)$. His analysis was restricted to a Newtonian ice rheology and utilized the non-separated flow results of Nye (1969) which assume slow sliding over a wavy bed with a relief of small slope. Hallet's analysis is appropriate to the case of a hard bed or bedrock bed.

The important element identified by Hallet (1979), which is the dominant cause of both basal debris drag and abrasion, is the flow of ice towards the bed and past ice-entrained rock fragments which are in bed contact. This phenomenon presumably occurs on stoss slopes (Hallet, 1981, p. 25) and is due to a combination of regelation and creep (Nye, 1969, equation (32)). Hallet's general approach to sparse debris drag and abrasion supplants that of Boulton (1974) which must be rejected.

Two models of debris drag and abrasion will be presented. First, an approach will be taken which is independent of Hallet's. Secondly, an approach similar to that of Hallet will be presented but which offers different results and conclusions than those obtained by Hallet. Both models will consider an idealized situation: plane non-separated flow over a hard sinusoidal wavy bed with equally spaced uniform spherical rock fragments entrained in the basal ice. Qualitative comparisons will then be made between these models and Hallet's. Generalizations of the models follow. Finally, a discussion of certain, possibly important, elements which are not considered here or in Hallet's theory will be presented.

\section{DEBRIS DRAG RELATED TO UNIFORM BASAL MELTING}

The first model considers the spatially averaged friction force exerted by the bed upon a rock fragment which makes periodic bed contact. It will be shown that it is unnecessary to know where rock-bed contact occurs. The important factor is the average melting rate of basal ice. Throughout, assumptions will be denoted by A1, A2, etc.

A1. A Newtonian ice rheology is assumed.

A2. The wavy bed is sinusoidal in the direction of ice flow with wavelength $\ell$.

A3. Assume that the slope of the bed roughness is sufficiently small that the sliding velocity may be expressed as $u_{\mathrm{b}}$, a constant, approximately equal to the $x$-component of sliding velocity (Fig. 1).

A4. Assume no ice-bedrock separation.

A5. Uniform spherical rock fragments of radius $R$ are equally spaced in the basal ice in a square array, distance $d$ apart where $d>2 R$ (Fig. 2).

A6. The abrasion of the rock fragments is neglected and no additional fragments are created by quarrying.

A7. The component of the fragment velocity relative to and parallel to the bed is assumed to be the ice-sliding velocity $u_{\mathrm{b}}$. Thus, the component of fragment velocity parallel to the bed and 
relative to the ice is neglected. (See Hallet (1981, p. 25) for amplification.)

A8. Periodic conditions, period $\ell$, exist for the ice flow, basal thermodynamics, and distance of the rock fragments from the bed. Thus, an individual rock fragment is in bed contact over a similar interval $x_{1} x_{2}$ (Fig. 1) for each wavelength of the bed. One needs to analyze the situation only for one particular bed wavelength.

A9. It is assumed that ice straining does not cause motion of the centre of a rock fragment relative to the ice when the fragment is not in bed contact.

A10. Neglect the settling velocity of ice of a rock fragment due to gravity when the fragment is not in bed contact.

A11. Neglect the force exerted by bedrock upon a fragment due to the difference in density between rock and ice, the buoyant weight.

None of these assumptions is inconsistent with Hallet's (1979, 1981) model, and Al, A3, A4, A6, A7, A9, A10, and A11 were either explicitly or tacitly assumed by him. Various of the assumptions will later be examined and some will be relaxed.

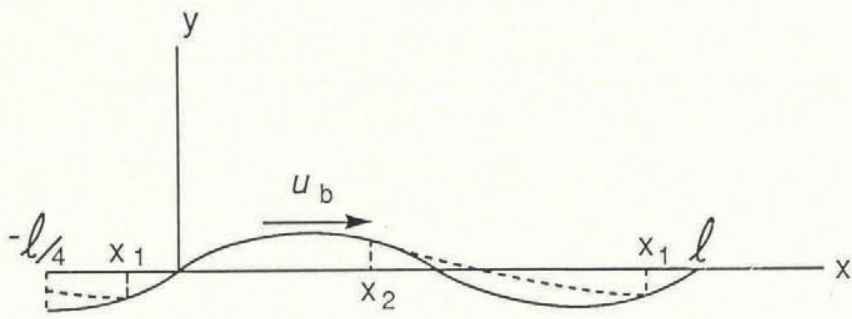

Fig. 1. Non-separated flow over a sinusoidal bed. A debris fragment is in bed contact on interval $x_{1} x_{2}$ and its base follows the dotted path outside this interval. Sliding velocity $u_{b}$ is uniform and time independent.

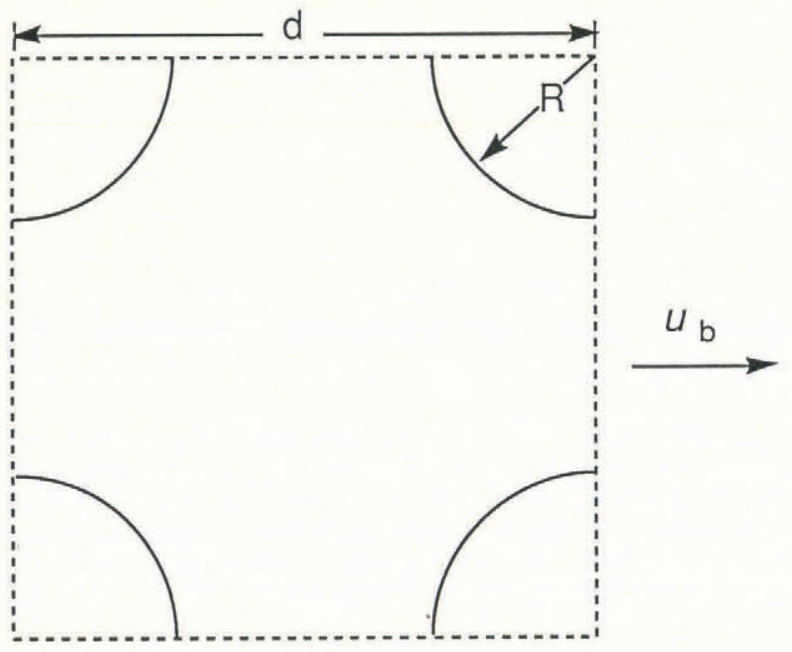

Fig. 2. Basal debris in a square array of uniform spheres. A convenient debris system is the four quarter-spheres within the dotted square.

Adopting the system rather than control volume point of view (Streeter and Wylie, 1981, p. 88), let $v_{a}$ be the temporal averaged melting rate at a point on the base of the ice as the point moves through wavelength distance $\ell$ relative to the bed. In the absence of basal debris and assuming basal ice of uniform properties, $v_{\mathrm{a}}$ would necessarily be constant over the bed because of the periodic conditions. With basal debris present, $v_{\text {a }}$ will likely not be uniform, primarily because of localized energy dissipation produced by rock-rock friction. This should increase the melting rate near basal debris fragments, an effect which was not considered by Hallet and will be neglected here.
A12. $v_{\mathrm{a}}$ is uniform over the base.

There are contributions to $v_{\mathrm{a}}$ from geothermal heating and what is usually termed sliding friction (Röthlisberger, 1968). As defined, $v_{\mathrm{a}}$ may be expressed as

$$
v_{\mathrm{a}}=\rho^{-1} H^{-1} \tau_{\mathrm{b}} u_{\mathrm{b}}+v_{\mathrm{g}}
$$

where $\rho$ is the density of ice, $H$ is the heat of fusion, $v_{\mathrm{g}}$ is the average melting rate over wavelength $\ell$ due to geothermal heat, and $\tau_{\mathrm{b}}$ is the average basal shear stress over $\ell$.

From assumptions $\mathrm{A} 6, \mathrm{~A} 8, \mathrm{~A} 9, \mathrm{~A} 10$, and $\mathrm{A} 12$, it follows that, over wavelength $\ell$, the average speed of penetration of a rock fragment into basal ice (or, equivalently, the average speed that ice moves past the fragment towards the bed) is $v_{\mathrm{a}}$. Although ice only flows past the fragment while the fragment is in bedrock contact, from the linearity implied by $A 1$ the average force exerted by bedrock upon the fragment, normal to the bed, as the fragment traverses a wavelength is

$$
\begin{gathered}
F=\Lambda_{\mathrm{R}} v_{\mathrm{a}}, \\
\Lambda_{\mathrm{R}}=\frac{f \pi 4 n R^{3}}{(R *)^{2}+R^{2}} .
\end{gathered}
$$

$\Lambda_{\mathrm{R}}$ was originally given by Watts (unpublished, p. 30 ); the introduction of a bed-influence factor $f$ was made by Hallet (1981, equation (2)). $\eta$ is the viscosity of basal ice and $R_{*}$ (Watts, unpublished, p. 30) is a transition radius analogous to the transition wavelength used in glacial sliding theory (Nye, 1969).

Equation (2) clearly shows the importance of the uniform basal melting rate $v_{\mathrm{a}}$. If $v_{\mathrm{a}}$ is assumed to be zero, there can be no rock-rock contact under the above assumptions. There could, however, be transient contact. Consider, for example, a rock fragment which is placed in bedrock contact on a stoss surface by an outside agency. The centre of the fragment would be pushed into the ice on the stoss surface. While traversing the subsequent lee surface, the fragment would be covered by regelation ice On a sinusoidal bed the fragment would never return to bedrock contact, assuming that $v_{\mathrm{a}}=0$. The condition $v_{\mathrm{a}}>0$ is necessary in order to return rock fragments to the bed after losing bedrock contact. This point will be amplified later.

By A5 we may define a basal debris concentration $C$ in units of projected area/unit basal area, as

$$
C=\pi R^{2} / d^{2}, 2 R<d .
$$

Note that $C$ is not a measure of debris actually in bedrock contact at any fixed time, but rather a measure of the debris which periodically contacts bedrock.

If $\mu$ is the coefficient of rock-rock friction, the debris-drag stress, $\tau_{\mathrm{d}}$, is

$$
\tau_{\mathrm{d}}=\frac{\mu F}{d^{2}}=\frac{\mu C F}{\pi R^{2}}=\frac{\mu \Lambda_{\mathrm{R}} C v_{\mathrm{a}}}{\pi R^{2}}
$$

having used Equations (4) and (2). Note that $\tau_{d}$ must be interpreted as being averaged over $\mathrm{a}$ bed wavelength and also averaged over time $\Delta t=\ell / u_{\mathrm{b}}$. On other length- and time-scales there are fluctuations in $\tau_{\mathrm{d}}$.

\section{DEBRIS DRAG ON INTERVALS OF ROCK-ROCK CONTACT}

Hallet (1979) approached debris drag by considering the average rock-rock contact force on stoss surfaces, assuming contact on the entire stoss surface. A more general approach of this nature is taken here with very different results than those obtained by Hallet.

Let the sinusoidal bed relief be given by

$$
y(x)=a \sin k x
$$


(Fig. 1), where $k=2 \pi / \ell$. Under assumptions A1, A2, A3, and $\mathrm{A} 4$, the $x$ and $y$ components of ice velocity, as given by Nye (1969, equation (32)), are

$$
\begin{aligned}
& u=u_{\mathrm{b}}+u_{\mathrm{b}} a \beta k^{2} y \mathrm{e}^{-k y_{\sin } k x} \\
& v=u_{\mathrm{b}} a \beta k(1+k y) \mathrm{e}^{-k y} \cos k x
\end{aligned}
$$

where

$$
\beta=k^{2}\left(k^{2}+k^{2}\right) .
$$

$k *$ was given by Nye (1969), p. 452) as $0.1 \mathrm{~cm}^{-1}$. By $\mathrm{A} 3$ and $\mathrm{A} 4$, it is assumed that $a k \ll 1$.

The component of ice velocity normal to the bed, positive if towards the bed, is from Equations (7) and (8)

$$
v_{\mathrm{n}}=u_{\mathrm{b}} a k\left[1-\beta(1+k y) \mathrm{e}^{-k y}\right] \cos k x+v_{\mathrm{m}}(x)
$$

where $v_{\mathrm{m}}(x)$ is the local contribution to the melting rate due to geothermal heat and sliding friction. In general, $v_{\mathrm{m}}(x)$ is not equal to $v_{\mathrm{a}}$ but they have the same average value over wavelength $\ell$, even if $\mathrm{A} 12$ is not invoked.

For simplicity of illustration, we will consider only the limiting case of regelation-dominant flow. This corresponds to $B \rightarrow 0$. For this case, Equation (10) reduces to

$$
v_{\mathrm{n}}=u_{\mathrm{b}} a k \cos k x+v_{\mathrm{m}}(x)
$$

and regelation melting on stoss surfaces alternates with refreezing on lee surfaces. The ice velocity normal to the bed is now independent of $y$. Provided $R$ is small compared with $\ell$, we may assume that the ice flow is uniform around the sphere when it is in bedrock contact and that the ice velocity towards the bed relative to the sphere may be computed from Equation (11) by letting $x$ be the coordinate of the centre of the sphere.

The interval $x_{1} x_{2}$ (Fig. 1), where a rock fragment is in bedrock contact, must now be determined. A necessary condition for contact is $v_{\mathrm{n}} \geqslant 0$. If $v_{\mathrm{n}}<0$, the center of the fragment is being carried away from the bed. Thus, point $x_{2}$ (Fig. 1), where rock-rock contact ceases, is determined by the condition

$$
v_{\mathrm{n}}\left(x_{2}\right)=u_{\mathrm{b}} a k \cos k x_{2}+v_{\mathrm{m}}\left(x_{2}\right)=0 .
$$

Since $v_{\mathrm{m}}(x)$ is difficult to determine, in order to exhibit the qualitative nature of the results, we make a final assumption.

$$
\text { A13. } v_{\mathrm{m}}(x)=v_{\mathrm{a}} \text {. }
$$

$x_{2}$ is then given by

$$
2 x_{2} / \ell=(1 / \pi) \cos ^{-1}\left(-v_{\mathrm{a}} \ell / 2 \pi u_{\mathrm{b}} a\right), \pi / 2 \leqslant \frac{2 \pi x_{2}}{\ell} \leqslant \pi .
$$

Point $x_{1}$ (Fig. 1), where the fragment re-establishes contact with the bed, is determined by the condition that the total distance that the fragment center is pushed into basal ice, while in bedrock contact on $x_{1} x_{2}$, equals $v_{\mathrm{a}} \mathrm{l} / u_{\mathrm{b}}$. Here, $\quad v_{\mathrm{a}} \ell / u_{\mathrm{b}}$ is the known penetration distance over wavelength $\ell$ calculated from the first model. Note that by A9 and A10 there is no motion of the fragment relative to the ice except on interval $x_{1} x_{2}$. Thus, $x_{1}$ is determined by the equation

$$
\int_{t\left(x_{1}\right)}^{t\left(x_{2}\right)} v_{\mathrm{n}}(x) \mathrm{d} t=v_{a^{\ell}} / u_{\mathrm{b}} .
$$

After substitution from Equation (11), and using A13, we obtain

$$
\frac{2 x_{1}}{\ell}+\frac{2 u_{\mathrm{b}^{a}}}{v_{\mathrm{a}} \ell} \sin \left[\frac{2 \pi x_{1}}{\ell}\right]=-2\left[1-\frac{x_{2}}{\ell}\right]+\frac{2 u_{\mathrm{b}} a}{v_{\mathrm{a}} \ell} \sin \left[\frac{2 \pi x_{2}}{\ell}\right] .
$$

It is easy to see from Equations (13) and (14) that both $x_{1} / \ell$ and $x_{2} / \ell$ depend only the parameter $v_{\mathrm{a}} \ell / 2 \pi u_{\mathrm{b}} a$.

Throughout the previous derivation we have made the tacit assumption that the flow field around the rock fragment while in bed contact does not alter the velocity field given by Equation (10). This assumption should be valid if $R \ll \ell$ but, if $R$ and $\ell$ are of the same order of magnitude, Equation (10) loses accuracy.

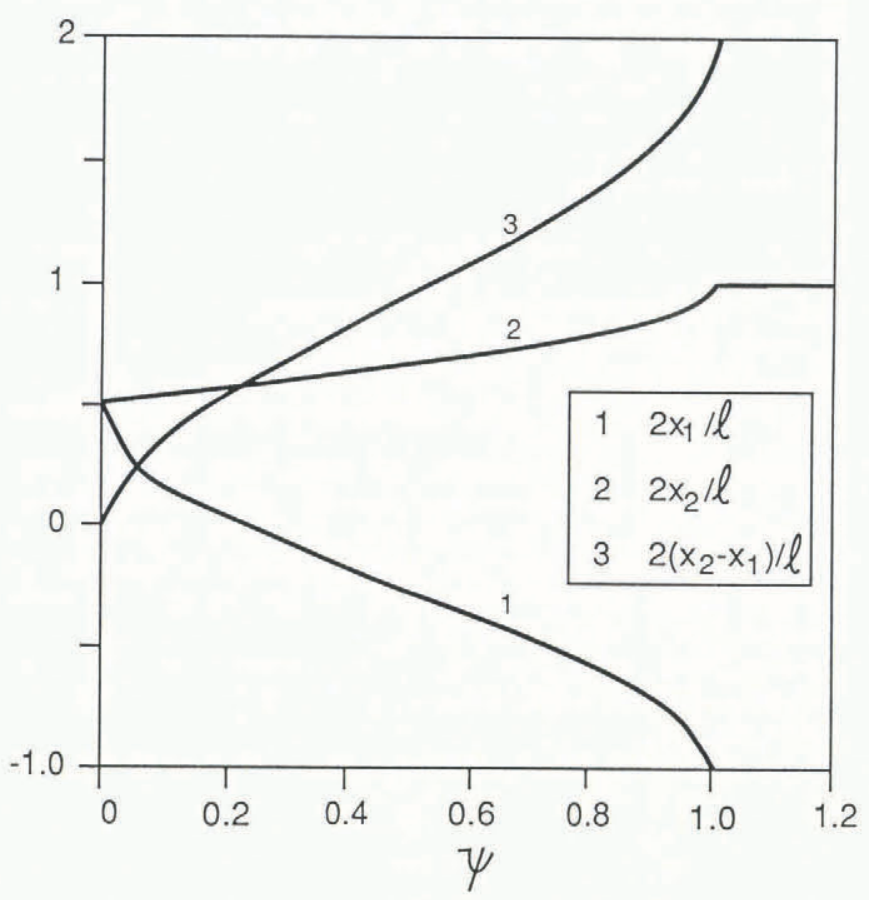

Fig. 3. $2 x_{1} / \ell, 2 x_{2} / \ell$, and $2\left(x_{2}-x_{1}\right) / \ell$ computed from Equations (13) and (14) versus $\psi=v_{a} l / 2 \pi u b_{b}$.

Figure 3 summarizes results for the variation of contact points $x_{1}, x_{2}$, and contact interval $x_{1} x_{2}$ as a function of parameter $\psi=v_{\mathrm{a}} \ell / 2 \pi u_{\mathrm{b}} a$. At the limiting state $\psi \rightarrow 0$, the contact interval reduces to a single point at $x=\ell / 4$ (Fig. 1). Since $u_{\mathrm{b}}$ is finite, this limiting state can only be attained if $v_{\mathrm{a}} \ell \rightarrow 0$. However, there is no physical basis for expecting that $v_{\mathrm{a}}$ can ever be zero. The condition $v_{\mathrm{a}} l=2 \pi u_{\mathrm{b}} a$ corresponds to a critical state. For this condition, Equation (13) gives $x_{2}=\ell / 2$ and Equation (14) gives $x_{1}=-\ell / 2$, so that rock-rock contact occurs everywhere. Equations (13) and (14) do not apply if $\psi>1$ but it is clear that $\left(x_{2}-x_{1}\right) / \ell=1$ as shown in Figure 3 .

Consider Figure 3 from the point of view that $C, \Lambda_{\mathrm{R}}$, and $v_{\mathrm{a}}$ are fixed with $a$ varying. Then $\tau_{\mathrm{d}}$ is fixed but the average force $\bar{F}$ over contact interval $x_{1} x_{2}$ is proportional to $1 /\left(x_{2}-x_{1}\right)$. Thus, as $a$ increases, $\boldsymbol{\psi}$ decreases towards zero and $\bar{F}$ increases.

Insight is gained by considering the balance of debris flux normal to the bed computed separately from the two models. Consider a basal debris system consisting of the parts of the four spheres which are inside the square shown in Figure 2. From the definition of $v_{\mathrm{a}}$, the temporal averaged flux of the system, positive if into the basal ice, of projected debris area per unit basal area is

$$
\Phi=v_{\mathrm{a}} C
$$

where $C$ is defined by Equation (4). The flux is measured relative to the bed. The contributions to $\Phi$ from individual rock fragments are positive over interval $x_{1} x_{2}$ and are nowhere negative in view of $A 9$ and A10. Therefore, $\Phi$ can also be computed from the second model by considering the flux of individual fragments over the interval of contact $x_{1} x_{2}$.

Let $\bar{v}_{\mathrm{nc}}$ be the average of $v_{\mathrm{n}}$ from Equation (11) over the contact interval $x_{1} x_{2}$. Then, $\Phi$ can be expressed in the form

$$
\Phi=\bar{v}_{\mathrm{nc}} C\left(x_{2}-x_{1}\right) / \ell
$$


where the factor $\left(x_{2}-x_{1}\right) / \ell$ is required because the flux is averaged over wavelength $\ell$, not over $\left(x_{2}-x_{1}\right)$. It is convenient to think of $C\left(x_{2}-x_{1}\right) / \ell$ as the basal debris concentration which is, on average, actually in contact with the bed. Thus, define

$$
C_{\mathrm{c}}=C\left(x_{2}-x_{1}\right) / \ell
$$

as the effective or contact-debris concentration. It is clear from Equations (17), (13), and (14) that $C_{\mathrm{c}}$ is not a constant but depends upon the parameter $\psi=v_{\mathrm{a}} l / 2 \pi u_{\mathrm{b}} a$.

Assuming that $v_{m}(x)=v_{a}$ in Equation ${ }^{(11),}$ it is possible to compute $\bar{v}_{\text {nc }}$ in Equation (16) by integrating Equation (11), assuming that $x_{1}$ and $x_{2}$ are known. Alternatively, $\bar{v}_{\text {nc }}$ may be obtained by equating Equations (15) and (16). Thus,

$$
v_{\mathrm{a}} \ell=\bar{v}_{\mathrm{nc}}\left(x_{2}-x_{1}\right) \text {. }
$$

By using Equation (17), this becomes

$$
v_{\mathrm{a}} C=\bar{v}_{\mathrm{nc}} C_{\mathrm{c}} .
$$

Equation (18b) is a statement of debris-flux balance. The term $\bar{v}_{\mathrm{nc}} C_{\mathrm{c}}$ is the debris flux directed into the ice on the contact interval $x_{1} x_{2}$. In magnitude, the term $v_{\mathrm{a}} C$ may be interpreted as the debris flux being returned to the bed by uniform basal melting. Note that, if $v_{\mathrm{a}} \ell / 2 \pi u_{\mathrm{b}} a>1$, both Equations (18) reduce to $v_{\mathrm{a}}=\bar{v}_{\text {nc }}$.

Equations (18) link the two models. We see, for example, that the debris-drag stress in Equation (5) may be written as

$$
\tau_{\mathrm{d}}=\frac{\mu \Lambda_{\mathrm{R}} C_{\mathrm{c}} \overline{\mathrm{v}}_{\mathrm{nc}}}{\pi R^{2}} .
$$

\section{COMPARISON WITH HALLET'S MODEL}

Hallet's $(1979,1981)$ approach is similar to the second model presented here. However, he assumed that there is rock-rock contact everywhere on stoss surfaces, regardless of the value of the parameter $v_{\mathrm{a}} \ell / 2 \pi u_{\mathrm{b}} a$. In fact, $v_{\mathrm{a}}$ is neglected in his analysis. Within the context of the regelation - only example considered here, a debris-drag stress predicted by Hallet's model could be obtained by setting $x_{2}-x_{1}=\ell / 2$, so that $C_{\mathrm{c}}=C / 2$ from Equation (17). When this value of $C_{c}$ is substituted into Equation (19) along with $\bar{v}_{\mathrm{ns}}$ in place of $\bar{v}_{\mathrm{nc}}$, we obtain

$$
\tau_{\mathrm{dH}}=\frac{\mu \Lambda_{\mathrm{R}} C \bar{v}_{\mathrm{ns}}}{2 \pi R^{2}} .
$$

Here, $\bar{v}_{\mathrm{ns}}$ is the average velocity normal to the bed in stoss surfaces with $\bar{v}_{\mathrm{m}}(x)$ deleted. obtain

Upon integrating Equation (11) from $-\ell / 4$ to $\ell / 4$, we

$$
\bar{v}_{\mathrm{ns}}=4 u_{\mathrm{b}} a / \ell
$$

and Equation (20) becomes

$$
\tau_{\mathrm{dH}}=\frac{2 \mu \Lambda_{\mathrm{R}} C u_{\mathrm{b}} a}{\pi R^{2} \ell} .
$$

The ratio of the debris-drag stress from Equations (5) and (22) is therefore

$$
\frac{\tau_{\mathrm{d}}}{\tau_{\mathrm{dH}}}=\frac{v_{\mathrm{a}} \ell}{2 u_{\mathrm{b}} a}=\frac{\left(\rho^{-1} H^{-1} \tau_{\left.\mathrm{b} u_{\mathrm{b}}+v_{\mathrm{g}}\right) \ell}\right.}{2 u_{\mathrm{b}} a}
$$

where Equation (1) was employed.

As a rough qualitative comparison of the debris-drag stresses predicted by Hallet and the present author, set $v_{\mathrm{g}}=0$ in Equation (23). Then, if $\tau_{\mathrm{b}}=10^{5} \mathrm{~Pa}$, we obtain $\tau_{\mathrm{d}} / \tau_{\mathrm{dH}}=1.6 \times 10^{-4} \ell / a$. The parameter $\ell / a$ is subject only to the restriction that $\ell / a \gg 1$. It is clear that $\tau_{\mathrm{d}} / \tau_{\mathrm{dH}}$ can have a wide range, from the order of $10^{-2}$ to values much greater than unity as a smooth bed is approached.

The physical reason for the lack of agreement between the two models can be understood with the aid of Figure 3. It is seen that if $\psi \ll 1$ then $2\left(x_{2}-x_{1}\right) / \ell \ll 1$. For this case, since Hallet assumed that $2\left(x_{2}-x_{1}\right) / \ell=1$, his model overestimates debris drag.

At the other extreme, as a smooth bed is approached and $\ell / a$ increases, $\psi$ eventually exceeds the critical value $\psi=1$. In the range $\psi \geqslant 1$, the rock fragments do not lose bed contact so that $2\left(x_{2}-x_{1}\right) / \ell=2$, instead of 1 as Hallet's model assumes. However, the primary reason why $\tau_{\mathrm{d}} / \tau_{\mathrm{dH}}>1$ for this case is that the influence of $v_{\mathrm{a}}$ dominates the influence of the cosine term in Equation (11) upon $\tau_{\mathrm{d}}$. But, Hallet's model does not include $v_{\mathrm{a}}$. (Note that, for this case, the cosine term in Equation (11) does not contribute to $\bar{v}_{\mathrm{nc}}$ and hence does not affect $\tau_{\mathrm{d}}$.)

Hallet's (1981, p. 26) conclusion that debris drag can slow glacier sliding should be re-examined. In Equations (3) and (5), we use Hallet's values of $\mu=1, f=2.4, \quad \eta=$ $3 \times 10^{12} \mathrm{Pas}$, and $R_{*}=0.1 \mathrm{~m}$. For fixed $v_{\mathrm{a}}$, drag is maximized for dense debris packing, $C=\pi / 4$ from Equation (4). (The exact form the model takes for densely packed particles is unknown. For the purpose of obtaining an upper bound on $\tau_{\mathrm{d}}$, it is appropriate to maximize $C$.) In addition, we choose $v_{\mathrm{a}}=1 \mathrm{~cm} \mathrm{a}^{-1}$ as a normalized value. $v_{\mathrm{a}}$ can be expected to lie in the range $1-11 \mathrm{~cm} \mathrm{a}^{-1}$ with the high value corresponding to a fast-sliding glacier $\sim 300 \mathrm{~m} \mathrm{a}^{-1}$ (Röthlisberger, 1968, p. 91). We also find that $\Lambda_{\mathrm{R}} / R^{2}$ is maximized if $R=R_{*}$. Equation (5) then gives an upper bound of $1.3 \times 10^{4} \mathrm{~Pa}$, corresponding to $v_{\mathrm{a}}=1 \mathrm{~cm} \mathrm{a}^{-1}$. We conclude that it is still possible for debris drag to be a significant component of total drag, particularly for fast-sliding glaciers.

Comparisons will now be made between abrasion rates predicted by the present models and those predicted by Hallet's (1981) model. We base the analysis upon Hallet's (1979, equation (2)) formulation

$$
\dot{a}=\alpha C_{\mathrm{r}}(\bar{x}) u_{\mathrm{b}} F(\bar{x})
$$

where $\dot{a}$ is a local abrasion rate (thickness of bedrock removed/unit time), $\alpha$ is an attritivity coefficient which will be taken as constant, and $u_{\mathrm{b}}$ the sliding velocity. $C_{\mathrm{r}} F$ is the product of $C_{r}$, the number of rock fragments in contact with the bed/unit area, and $F$, the average force normal to the bed exerted on these fragments which are included in the calculation of $C_{\mathrm{r}}$. (We have taken the liberty of replacing fragment velocity (Hallet, 1979, equation (2)) by sliding velocity. See Hallet $(1979,1981)$ for justification.)

One comparison which will not be made is the effect of differences between $\tau_{\mathrm{d}}$ and $\tau_{\mathrm{dH}}$ upon abrasion rates. Clearly, if $u_{\mathrm{b}}$ in Equation (24) is sensitive to debris drag, i.e. debris drag dominates conventional drag, Hallet's and the present model could predict very different abrasion rates, depending upon the value of $\psi$. However, I take the view that not enough is known of either conventional drag or debris drag to warrant quantitative comparisons of the effect of debris-drag formulations upon abrasion rates.

It remains to compare abrasion-rate models, given that $u_{\mathrm{b}}$ in Equation (24) is independent of which model is being considered. We first compare abrasion rates averaged over a sinusoidal bed. A spatially averaged abrasion rate $\dot{A}$ may be obtained, using the first model developed above, if $C_{\mathrm{r}}$ in Equation (24) is consistent with $C$ defined by Equation (4), and $F$ is as in Equation (2). For the square array of uniform spherical debris fragments we therefore have

$$
C_{\mathrm{r}}=C / \pi R^{2} \text {. }
$$

Substituting from Equations (25) and (2) into Equation (24), with $\dot{a}$ replaced by $\dot{A}$, gives

$$
\dot{A}=\frac{\propto u_{\mathrm{b}} C}{\pi R^{2}} \Lambda_{\mathrm{R}} v_{\mathrm{a}} .
$$


As in the formulation of basal debris drag, the present approach diverges from Hallet's model. If his model is used to formulate the average abrasion rate $\dot{A}$, the result, from a similar analysis which led to Equation (26), would be

$$
\dot{A}_{\mathrm{H}}=\frac{\propto u_{\mathrm{b}} C}{2 \pi R^{2}} \Lambda_{\mathrm{R}} v_{\mathrm{ns}}
$$

where $\bar{v}_{\text {ns }}$ is given by Equation (21) for regelationdominated flow. From Equations (26) and (27), we obtain

$$
\frac{\dot{A}}{\dot{A}_{\mathrm{H}}}=\frac{\ell v_{\mathrm{a}}}{2 a u_{\mathrm{b}}}=\pi \psi .
$$

It must be noted that Hallet did not compute a spatially averaged abrasion rate. His numerical results (Hallet, 1981) are apparently confined to the local abrasion rates, $\dot{a}_{\mathrm{S}}$, at the centre of stoss surfaces. It is possible to show that the present model gives results for $\dot{a}_{\mathrm{S}}$ which agree with Hallet's, provided that the debris-drag component is dominated by conventional drag, i.e. provided that $u_{\mathrm{b}}$ in Equation (24) is the same for both models.

The abraded region is confined to the interval $x_{1} x_{2}$ of rock-rock contact. In general, the entire stoss surface is not abraded. The local abrasion-rate pattern on interval $x_{1} x_{2}$ may easily be found for a sinusoidal bed by utilizing the second model developed above. This will not be done but we note that the average abrasion rate over $x_{1} x_{2}$ is given by $\left(\ell /\left(x_{2}-x_{1}\right)\right) \dot{A}$, where $\dot{A}$ is given by Equation (26).

Hallet's (1981, figs 3, 4, and 5) results indicate a dependence of local abrasion rate upon bed wavelength. This conclusion is probably true for the case he considered, the local abrasion rate at, say, the center of a stoss surface. However, it is clear from Equation (26) that there is no explicit dependence of average abrasion rate $\dot{A}$ upon $\ell$ and no dependence whatever provided $\tau_{\mathrm{b}}$ and $u_{\mathrm{b}}$ are held constant.

\section{GENERALIZATIONS}

1. With the removal of the restriction that the ice flow over the sinusoidal bed is dominated by regelation, $B$ in Equation (9) enters as a parameter in the second model. $B$ has the range $[0,1]$ with $B=0$ corresponding to regelation - only flow and $\beta=1$ corresponding to flow by creep. Because, for given $v_{\mathrm{a}}, \beta$ does not enter the first model, the (average) debris drag $\tau_{\mathrm{d}}$ and average abrasion rate $\dot{A}$ are unchanged. Note that parameter $\Lambda_{\mathrm{R}}$ in Equations (3) and (5) is affected by the relative importance of the regelation and creep mechanisms for flow around rock fragments when in bed contact. Thus, the ratio $R / R *$ influences $\tau_{\mathrm{d}}$.

If Equation (10), which contains $B$, is used in place of Equation (11) in the development of the second model, $x_{1}$ and $x_{2}$ will depend upon $\beta$ as well as $\psi$. A third parameter also enters since, from Equation (10), the flow around a sphere in bed contact depends upon coordinate $y$. If Hallet's (1981) procedure is followed, $v_{n}$ in Equation (10) is evaluated at $y=R$, the center of the sphere. Thus, $x_{1}$ and $x_{2}$ are dependent upon $\psi, \beta$, and $R / R_{*}$. The development of this analysis will not be presented here.

2. The only assumptions actually used in developing the first model were A1, A3, A6, A7, the periodicity part of A8, A9, A10, A11, and A12. Thus, the model would appear to apply, without modification, to separated plane flow over non-sinusoidal but periodic beds with a single period.

3. A case can be made that the first model can also be applied to an arbitrary three-dimensional wavy bed. Consider a flow-line segment of sufficient length such that average values of $\tau_{\mathrm{b}}, u_{\mathrm{b}}$, and $v_{\mathrm{g}}$ are either known from field studies or can reasonably be assigned. Then, an average value of $v_{\text {a }}$ can be computed from Equation (1). Using reasonable estimates of $C, v_{\mathrm{g}}$, and $\Lambda_{\mathrm{R}}$, average values of $\tau_{\mathrm{d}}$ and $\dot{A}$ can then be computed from Equations (5) and (27), respectively.
4. Generalizations are not as simple if we are interested in studying local abrasion patterns, since intervals of rock-rock contact can be difficult to determine, particularly if separated flow is included. However, the generalization to plane non-separated flow over a non-sinusoidal periodic bed with a single period is straightforward. One need only use more terms from Nye's (1969) equation (32) in place of Equation (10).

It should be recognized that the effective debris concentration $C_{\mathrm{c}}$ on a particular bedrock bump of an arbitrary wavy bed depends not only upon the geometry of the particular bump but also upon the geometry of up-stream bumps. For example, a particular bump could act to cover rock fragments with ice of sufficient thickness that no bed contact would occur down-stream on smaller bumps, until several had been traversed.

5. One generalization which will be investigated is the effect of removing $\mathrm{A} 10$ and A11. The settling velocity $U$ and buoyancy force $F_{\mathrm{B}}$ should be considered together, because both result from $\left(w_{r}-w_{\mathrm{i}}\right)$, the difference in the specific weights of rock and ice

The appropriate change to the first model is simply the addition of the term

$$
F_{\mathrm{B}}=4\left(w_{\mathrm{r}}-w_{\mathrm{i}}\right) \pi R^{3} / 3
$$

to the right-hand side of Equation (2). The settling velocity does not then enter into the first model. To evaluate the importance of $F_{\mathrm{B}}$, we consider the ratio $W_{\mathrm{B}} / F_{1}$ where $F_{1}$ is computed from Equation (2) using $v_{\mathrm{a}}=1 \mathrm{~cm} \mathrm{a}^{-1}$, as a typical low value of $v_{\mathrm{a}}$. Table I gives $W_{\mathrm{B}} / F_{1}$ values based upon $\left(w_{\mathrm{r}}-w_{\mathrm{i}}\right)=10^{4} \mathrm{~N} \mathrm{~m}^{-3}, \eta=3 \times 10^{\mathrm{B}} \mathrm{Pa} \mathrm{s}$, and $R_{*}=$ $0.11 \mathrm{~m}$. It is clear that the buoyancy force only becomes important for large clasts.

TABLE I. RATIOS $W_{\mathrm{B}} / F_{1}$ AND $W_{\mathrm{B}} / F_{2}$ FOR VARIOUS $R$ VALUES $(\mathrm{m})$

\begin{tabular}{lll}
\multicolumn{1}{c}{$R$} & $W_{\mathrm{B}} / F_{1}$ & $W_{\mathrm{B}} / F_{2}$ \\
0.01 & 0.018 & 0.032 \\
0.1 & 0.032 & 1.47 \\
1 & 1.48 & 146
\end{tabular}

There is some evidence that the viscosity of debris-rich basal ice could be up to two orders of magnitude lower than for ordinary glacier ice (Echelmeyer and Zhongxiang, 1987). Part of the effect could be due to the increased water content of ice near the bed (Duval, 1977). Using $v_{\mathrm{a}}=1 \mathrm{~cm} \mathrm{a}^{-1}, \quad \eta=3 \times 10^{10} \mathrm{Pas}$, and $R *=0.011 \mathrm{~m}$ (Watts, unpublished, equation (23)) to compute $F_{2}$ from Equation (2), we determine the ratios $W_{\mathrm{B}} / F_{2}$ shown in Table I. Noting that conventional drag is proportional to $n$, it is apparent that $W_{\mathrm{B}}$ could be important for moderate to large clasts, particularly for soft basal ice.

Both $U$ and $W_{\mathrm{B}}$ enter into the second model. Contact points $x_{1}, x_{2}$ are affected and $W_{\mathrm{B}}$ is added to the contact force over interval $x_{1} x_{2}$. The analysis is straightforward and will not be presented.

\section{POSSIBLE IMPORTANT FACTORS NOT INVESTIGATED}

There are at least two remaining potentially important influences on debris drag, both of which are difficult to analyze. The first, previously noted, is the effect of rock-rock friction in causing localized melting near debris fragments. This will serve to increase melting rates near rock fragments and thus increase $\tau_{\mathrm{d}}$. I believe that this effect will prove to be significant.

The second influence upon $\tau_{\mathrm{d}}$ brings $\mathrm{A} 9$ into question. It is possible to show that on a stoss face a rock fragment not in bedrock contact will tend to be moved away from the bedrock by the action of the strain field. On a lee face, the opposite effect occurs. However, it is not clear that the two effects cancel because, for separated flow, the boundary conditions are very different on the two faces. I have been unable to solve this problem for realistic boundary conditions. I can only identify the ejection of rock fragments from the ice face in separated flow on lee surfaces as being potentially important. 


\section{CONCLUSIONS}

The two models of debris drag and bedrock abrasion developed here are formulated for the easily understood case of non-separated flow on a sinusoidal bed. Generalizations to arbitrary beds are possible for the first model and a certain degree of generalization is possible for the second one. The critical role of $v_{\text {a }}$ (commonly called uniform basal melting), in terms of which the first model is formulated, has been established. Consistent inter-relationships between the two models have been emphasized. Quantitative comparison between debris drag and conventional drag have been avoided; such numbers are probably meaningless at this stage.

Referees objections to the contrary, I believe that it has been established that the debris concentration $C$ is more natural to deal with than $C_{\mathrm{C}}$, which depends upon parameter $\psi$ for a sinusoidal bed but would have a much more complex dependency for an arbitrary bed. At least for theoretical studies, it is easier to assign reasonable values to $C$, a constant, than it is to deal with $C_{C}$ and its complex functional dependency. Not do I agree that in field work it would be easier to determine $C_{\mathrm{c}}$ than $C$.

\section{REFERENCES}

Boulton, G.S. 1974. Processes and patterns of glacial erosion. In Coates, D.R., ed. Glacial geomorphology. Binghamton, NY, State University of New York, 41-87.

Duval, P. 1977. The role of the water content on the creep rate of polycrystalline ice. International Association of Scientific Hydrology Publication 118 (General Assembly of Grenoble 1975 - Isotopes and Impurities in Snow and Ice), 29-33.

Echelmeyer, K. and W. Zhongxiang. 1987. Direct observation of basal sliding and deformation of basal drift at sub-freezing temperatures. J. Glaciol., 33(113), 83-98.

Hallet, B. 1979. A theoretical model of glacial abrasion. $J$. Glaciol., 23(89), 39-50.

Hallet, B. 1981. Glacial abrasion and sliding: their dependence on the debris concentration in basal ice. Ann. Glaciol., 2, 23-28.

Nye, J.F. 1969. A calculation on the sliding of ice over a wavy surface using a Newtonian viscous approximation. Proc. R. Soc. London, Ser. A, 311, 445-467.

Röthlisberger, H. 1968. Erosive processes which are likely to accentuate or reduce the bottom relief of valley glaciers. International Association of Scientific Hydrology Publication 79 (General Assembly of Bern 1967 - Snow and Ice), 87-97.

Streeter, V.L. and E.B. Wylie. 1981. Fluid mechanics. New York, McGraw-Hill.

Watts, P.A. Unpublished. Inclusions in ice. (Ph.D. thesis, University of Bristol, 1974.)

MS. received 12 July 1985 and in revised form 13 July 1988 